\title{
Second-order IIR Notch Filter Design and implementation of digital signal processing system
}

\author{
Chun Mei WANG \\ Guangdong University of Finace \\ Guangzhou, China \\ mei_wangchun@163.com
}

\author{
Wei Cai XIAO \\ Guangdong University of Finace \\ Guangzhou, China \\ shlbdzdsj@163.com
}

\begin{abstract}
In this paper the AC power $50 \mathrm{~Hz}$ power interference, we use IIR digital notch filter method for industrial frequency interference filter. From the design of IIR digital filter method proceed with, on the IIR digital notch filter simulation, the algorithm deduced, on the fixed-point DSP programming method and overflow handling problems made elaborate incisively, and in digital audio signal processing system has been applied.
\end{abstract}

\section{Keywords- IIR;Notch Filter;Z transform}

\section{INTRODUCTION}

Many occasions by a digital filter to filter out certain frequency noise, for example, in the detection of weak signal, often have $50 \mathrm{~Hz}$ power interference signal, and as in audio amplifier speakers close to the microphone, will produce a self feedback whistle signal. These harmful noise signal is harmful, while retaining the original signal at the same time, how to eliminate interference, the main purpose is to design a filter ${ }^{[1]}$. System structure is shown in figure 1.

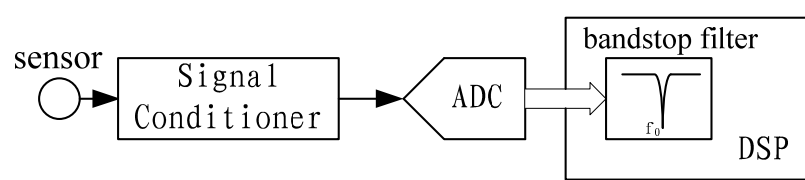

Figure 1. typical applications of digital notch filter

If the filter specified frequency filter is actually a bandstop filter, also known as a notch filter. Design of band-pass filter method, design of notch filter method has a lot of, usually available all-pass filter minus band pass filter ${ }^{[2][3]}$. In this paper, using two order $\mathrm{Z}$ transfer function pole zero placement method of direct design of two order IIR notch filter. This method is very effective and practical.

If you want to achieve high order filter or on multiple frequency notch, can use a plurality of two order wave trap cascade.

\section{SECOND-ORDER IIR DIGITAL NOTCH FILTER DESIGN}

Notch filter is of certain frequency of the signal are eliminated, and the other components of the signal is not affected $^{[4]}$. Therefore, an ideal notch filter frequency response:

$$
H\left(e^{j \omega}\right)= \begin{cases}0, & \omega_{0} \\ 1, & \text { otherwise }\end{cases}
$$

Wherein, $\omega 0$ notch filter center frequency.

A. two order wave trap $Z$ transfer function

the second order system of IIR digital filter of the $\mathrm{Z}$ transfer function:

Second-order system function:

$$
H(z)=\frac{\left(z-e^{j \omega_{0}}\right)\left(z-e^{-j \omega_{0}}\right)}{\left(z-r e^{j \omega_{0}}\right)\left(z-r e^{-j \omega_{0}}\right)}
$$

In the formula, $e^{j \omega_{0}}$ and $e^{r j \omega_{0}}$ respectively for the trap of zeros and poles. Select the unit circle for the frequency domain of a cycle, in order to prevent the filter coefficients appear complex, must be in plane fourth quadrantal symmetry position in response to the conjugate zero $e^{-j \omega_{0}}$ and $r e^{-j \omega_{0}}$ conjugate poles. The pole placement in zero radial distance from the origin of $\mathrm{R}$, therefore $0 \leq \mathrm{r}<1$. $\mathrm{R}$, pole is close to the unit circle, the frequency response in deeper, narrower in width.

In the zero point, frequency response appeared minima, said system " Valley ", in the pole frequency response has a maximum value, the " peak " point representation system. Valley point frequency cutoff, peak frequency through. According to the frequency response of the zero and pole allocation, the reverse design of notch filter. Let $\mathrm{z}=e^{j \omega}$, when $\omega=\omega_{0}$, infinite attenuation

Using Euler formula $\mathrm{e}^{\mathrm{j} \mathrm{x}}=\cos \mathrm{x}+\mathrm{j} \sin \mathrm{x}$ ( 2) type is simplified as

$$
H(z)=\frac{1-2 \cos \omega_{0} z^{-1}+z^{-2}}{1-2 r \cos \omega_{0} z^{-1}+r^{2} z^{-2}}
$$

\section{B. IIR notch filter simulation}

For the (3) type, you can use MATLAB simulation design.

For example, the design of a center frequency of $\mathrm{f}_{0}=50 \mathrm{~Hz}$ notch filter, filter the AC power line interference. Effective signal maximum frequency of $300 \mathrm{~Hz}$, and a sampling frequency $\mathrm{f}_{\mathrm{s}}=600 \mathrm{~Hz}, \mathrm{r}=0.99$, MATLAB simulation procedure is as follows:

$\mathrm{fs}=1000 ; \mathrm{fO}=50$;

b0 $=1$; 
$\mathrm{b} 1=-2 * \cos (2 * \mathrm{pi} * \mathrm{f} 0 / \mathrm{fs})$

b2 $=1$;

$\mathrm{r}=0.999$;

$\mathrm{a} 0=1$

$\mathrm{a} 1=-2 * \mathrm{r} * \cos (2 * \mathrm{pi} * \mathrm{f} 0 / \mathrm{fs})$

$\mathrm{a} 2=\mathrm{r} * \mathrm{r}$;

$\mathrm{b}=[\mathrm{b} 2, \mathrm{~b} 1, \mathrm{~b} 0]$;

$\mathrm{a}=[\mathrm{a} 2, \mathrm{a} 1, \mathrm{a} 0]$;

$[\mathrm{h}, \mathrm{w}]=$ freqz $(\mathrm{b}, \mathrm{a})$;

$\mathrm{hf}=\mathrm{abs}(\mathrm{h})$;

figure(1);

$\operatorname{plot}\left(\mathrm{w}^{*} \mathrm{fs} /(2 * \mathrm{pi}), \mathrm{hf}\right)$;

title('Magnitude Response');

xlabel('Freguency in $\mathrm{Hz}$ ');

figure(2);

zplane(b,a);legend('zero','plot');

Simulation of the amplitude frequency characteristic and

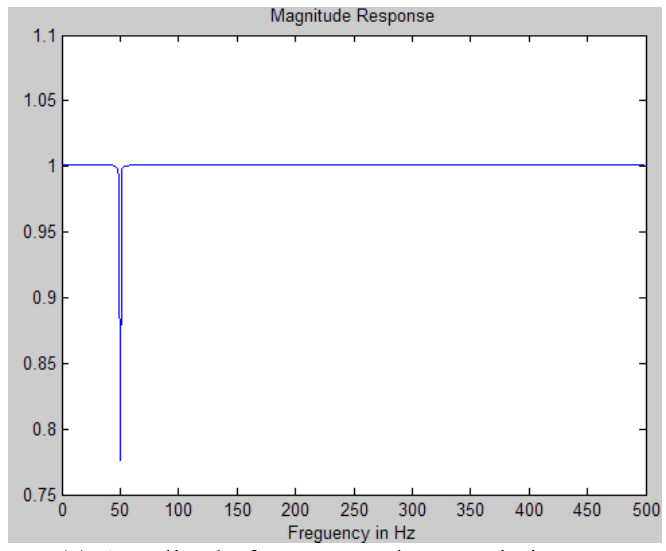

(a) Amplitude frequency characteristic

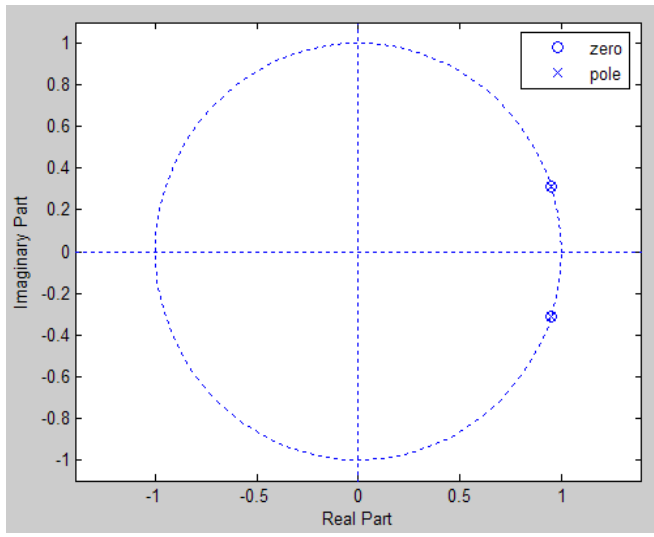

(b)pole-zero location

system of pole-zero diagram as shown in figure 2.

Figure 2. digital notch filter frequency characteristics and the distribution of zeros and poles
If the $\mathrm{H}(\mathrm{z})$ is multiplied by a coefficient $\mathrm{k}, \mathrm{k}$ is the amplification factor of the notch filter, which change the gain of the notch filter.

\section{Multi frequency digital notch filter}

If $\mathrm{H}$ ( $\mathrm{z}$ ) is multiplied by a factor of $\mathrm{K}, \mathrm{K}$ notch filter amplification coefficient, this changes the notch filter gain.

The IIR filter is a single frequency wave trap, when $n$ $(\mathrm{n}>1)$ frequency at the same time to notch, according to (2) configuration of different center frequency wave trap, then $n$ notch filter connected in series, as shown in figure 3.

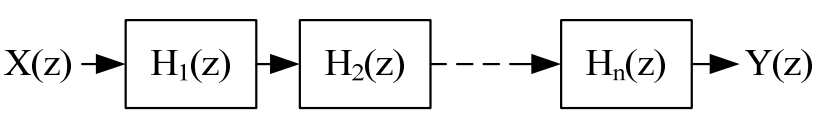

Figure 3. notch filter series

Two order IIR trap likely pole can not meet the requirements, the zero point determines the center frequency, cannot be changed ${ }^{[5][6]}$. But for the pole can be changed by type:

$$
H(z)=\frac{1-2 \cos \omega_{0} z^{-1}+z^{-2}}{1-2 r \cos \left(\omega_{0} \pm \phi\right) z^{-1}+r^{2} z^{-2}}
$$

Type of $\varphi$ is constant, is deviated from the zero pole in the radial direction.

\section{IIR FILTER ALGORITHM AND DSP REALIZATION}

For a two order digital filter of the $\mathrm{Z}$ transfer function can be expressed as:

$$
H(z)=\frac{Y(z)}{X(z)}=\frac{b_{0}+b_{1} z^{-1}+b_{2} z^{-2}}{1-a_{1} z^{-1}-a_{2} z^{-2}}
$$

In the formula, A1, A1, B, B1 and $\mathrm{B} 2 \neg 0$ can be expressed as coefficient, input / output output relation of linear difference equations with constant coefficients:

$$
y(n)=a_{1} y(n-1)+a_{2} y(n-2)+b_{0} x(n)+b_{1} x(n-1)+b_{2} x(n-2)
$$

Thus, the $\mathrm{N}$ output with the 2 previous output value $\mathrm{Y}$ $(\mathrm{n}-1), \mathrm{y}(\mathrm{n}-2)$ and the $\mathrm{N}$ input value $\mathrm{x}(\mathrm{n})$ and to the two input value $x(n-1), X(n-1)$ about.

This is a multiplication and addition operations of polynomial, DSP to achieve the fast calculation of polynomial, often provide polynomial for the calculation of the multiply-add instructions. At present, most of the DSP device is a fixed-point arithmetic device, but in the filter $\mathrm{Z}$ in transfer function coefficient for floats, in order to make the fixed-point DSP implementation of floating-point operations, there are two kinds of the following methods:

(1) preparation of floating point multiply, add operator program, to deal with floating point arithmetic problem;

(2) to ( 6) type coefficients in the A1, A1, B, B1, B2 $\neg 0$ magnification of $\mathrm{K}$, make the amplified coefficient of integral after meet the required precision, and then itemized integer multiplication, addition, $\mathrm{KY}(\mathrm{n})$, finally be divided by $K, y(n)$. Here the best choice of $k=2 i(i=1,2,3, \ldots)$ So, 
the coefficient of amplification, 2I times, can use the left shift coefficient shift left I to solve, in order to avoid multiplication. Similarly, in the narrow 2I times, can use the right shift instruction will result right shift left I bits to solve, in order to avoid a division operation.

The first method has the advantages of higher precision, but the floating point arithmetic program complex, long execution time. The second kind of method is simple, short execution time, is especially suitable for real-time processing, but has limited precision, and is easy to overflow. Fixed point DSP to point operations, will enlarge the value of a polynomial, if the data operation of the intermediate results are not big enough storage unit, will inevitably lead to overflow. Analysis of the differential algorithm polynomial shows, the main reason lies in the overflow system poles, namely system feedback link. Therefore, to calculate the likely maximum with signed numbers, to determine the maximum storage unit.

For example, the coefficient $\mathrm{A} 1, \mathrm{~A} 1, \mathrm{~B}, \mathrm{~B} 1, \mathrm{~B} 2 \neg 0$ magnification 2I times to sufficient accuracy, and in 32 signed binary number using the complement representation, data value indicates that the range of $-231 \sim(+231-1)$, the sampling input signal $x(n), X(n-1), X(n-2)$ are for the 16 binary digits, with 32 for 16 , will be extended to the sign bit, two 32 digit numbers, in order to avoid the overflow of intermediate results need 64 unit to store. In addition, when available 64 to complete, complete a polynomial operations, will result in reduced 2I times, get y ( $\mathrm{n})$, and in 32 signed to storage, and a check for overflow.

\section{HIGH ORDER FILTER BASED ON IIR}

At the request of filter has better properties, such as notch filter requires a particular bandwidth, notch depth and other conditions, can be made of high order filter based on IIR to achieve, if directly from high order filter transfer function derived $\mathrm{Z}$ difference equation, the signal delay will be longer ${ }^{[7]}$. From theoretic say, can use the high order IIR digital filter to achieve a good filtering effect. But DSP itself finite word length and accuracy factors, plus the IIR filter in the structure of the existing feedback loop, is a recursive type, and high order filter parameters of large dynamic range $\left[{ }^{8][9]}\right.$. Thus causing two consequences : results of overflow and the error is increased, thereby causing the algorithms can not achieve in DSP.

The effective way to solve this problem is to high order IIR digital filter is simplified as several 2 order filter design, which adopts the cascade structure. So the system function:

$\mathrm{H}(\mathrm{z})=\mathrm{H} 1(\mathrm{z}) \mathrm{H} 2(\mathrm{z}) \ldots \mathrm{Hn}(\mathrm{z})$
At each level of the local filter Hn ( $\mathrm{z}$ ) is one such as (5) the two order filter.

\section{CONCLUSIONS}

IIR digital notch filter with its superior performance, in a digital processing system to be widely applied. This paper describes the IIR digital notch filter design theory and implementation in DSP process. According to the train of thought, can be easily designed to meet the requirements of the filter. The method is very practical, especially for the realization of embedded systems have a very good reference value. Through the " digital signal processing " system in the notch filter design and the realization that the design method is simple, convenient, flexible, implementation, to meet the actual needs of system.

Theory and practice prove, digital audio signal processing system using IIR digital notch filter, AC power supply $50 \mathrm{~Hz}$ power interference eliminated does not affect the effective audio signal transmission, performance improvement, the signal-to-noise ratio can be improved greatly. The final results show that, the proposed method is simple, practical, effective.

\section{REFERENCES}

[1] H.B,Ju,Research and design based on notch filter active power filter [D]; Shandong University; 2010

[2] R.Jiang,Research and Design of airborne low frequency narrowband filter [D]; Henan Polytechnic University; 2011;

[3] B.Peng,Multi-channel linear phase uniform filter bank and the approximate wavelet filter bank [D]; Xi'an University of Electronic Science and Technology; 2009;

[4] Z.Z.Yang,A fractional differential IIR filter design methods and improvements [J]; modern electronic technology, 2009,32 (2) :54-58.

[5] SONG Yi-jun, LI Wen-yuan, SONG Yao-liang.The fractional IIR and FIR filter and their inverse systems[J]. JOURNAL OF CIRCUITS AND SYSTEMS,2011,16(6)41-45.

[6] Hypo-sung A,Varsga B,Yangquan C. Fractional-order Intergral and Derivative Controller for Temperature Profile Tracking [J].Sadahana,Academy Proc . in Eng..India,2009,34(5):833-850.

[7] CHEN Min-jiang,ZHAO Shu-yin,WU Zi-long. Applications of the Z Transformation . JOURNAL OF LANGFANG TEACHERS COLLEGE,2009,9(1): 27-29.

[8] HUANG X,CHU J,LO W,CUI H,WANG Z H.Simplified Method of Designing FIR Filter with Controllable Center Frequency.Transactions of Tianjin University Vol.16 No.4,pp262266,2010 .

[9] LAI X P, ZHAO R J.Minimax Design of Time-domain Constrained FIR Filters by a Sequential Constrained Least-Square Method.Chinese Journal of Electronics,Vo1.18,No.1,pp.166170,2009 . 